\title{
A probabilistic and interactive decision-analysis system for unruptured intracranial aneurysms
}

\author{
Narcyz Ghinea, B.Sc. (Hons), ANd James M. van Gelder, M.D. \\ Departments of Neurosurgery, South Western Sydney Area Health Service, University of New South \\ Wales; and Liverpool Health Service, Liverpool, New South Wales, Australia
}

\begin{abstract}
Object. The goal in this study was to develop an interactive, probabilistic decision-analysis system for clinical use in the decision to treat or observe unruptured intracranial aneurysms. Further goals were to enable users of the system to adapt decision-analysis methods to individual patients and to provide a tool for interactive sensitivity analysis.

Methods. A computer program was designed to model the outcomes of treatment and observation of unruptured aneurysms. The user supplies probabilistic estimates of key parameters relating to a specific patient and nominates discount rate and quality of life adjustments. The program uses Monte Carlo discrete-event simulation methods to derive probability estimates of the outcomes of treatment and observation. Results are expressed as summary statistics and graphs. Discounted quality-adjusted life years are graphed using survival methods. Hierarchical simulations are used to enable investigators to perform probabilistic sensitivity analysis for one or multiple parameters simultaneously. The results of sensitivity analysis are expressed in graphs and as the expected value of perfect information. The system can be distributed and updated using the Internet.

Conclusions. Further research is required into the benefits of clinical application of this system. Further research is also required into the optimum level of complexity of the model, into the user interface, and into how clinicians and patients are likely to interpret results. The system is easily adaptable to a range of medical decision analyses.
\end{abstract}

KEY WORDS - intracranial aneurysm • decision support • sensitivity analysis

The decision to treat an unruptured intracranial aneurysm involves a tradeoff between the immediate hazard and expense posed by treatment and the long-term risk of rupture. These decisions are unlikely to be sufficiently addressed by controlled trials for several reasons: there are few if any such trials in progress, the long duration of such trials compared with the rate of development of new techniques, and difficulty in generalizing the results of trials to individual patients with their numerous clinical details.

Decision analysis is a formalized, quantitative approach to clinical decision making. The possible outcomes of each alternative are listed, the probabilities of these outcomes are estimated, values are assigned to the outcomes, and the decision alternatives are compared in terms of a common utility. Selecting and combining the most relevant and reliable information is difficult and laborious. Numerous medical decision-analysis studies have been published, including several addressing the decision to treat unruptured aneurysms. ${ }^{1,2,5,6,10,12,13,15,20,22,32}$

The decision analyses available provide useful guidelines but have limitations when applied to individual patients. These limitations include the following. ${ }^{7}$

Abbreviations used in this paper: $\mathrm{CI}=$ confidence interval; DQALY = discounted quality-adjusted life year; EVPI = expected value of perfect information; QOL = quality of life.
1) The analysis is performed in a limited set of base case scenarios. Limited sensitivity analysis is provided but it often addresses just a few factors and limited ranges. It is often impossible to extrapolate the results of the analysis to individual cases. 2) The data used for the analysis may be inadequate. Important new information may have become available. The data selection may be controversial, incomplete, or have been subjected to insufficient critical appraisal. Expert opinion may be controversial or not locally applicable. 3) The form of the results (for example, a change in DQALYs) may not adequately address the concerns of clinicians and patients. Patients may want more details about the expected outcomes. Individual perceptions and preferences about the life expectancy, risk aversion, and the QOL with disabilities may need to be addressed with more descriptive results of the analysis. The discount rate for future life years can be easily misunderstood or misapplied. 4) The available decision analyses usually incorporate point estimates for some or all of the input parameters (deterministic models). Therefore, uncertainties in the available data may be ignored, causing false statements of precision in the results. The alternative is to use probabilistic analysis models that incorporate uncertainty in the input parameters. Probabilistic models give a more realistic description of the uncertainty in the results that arises from limitations in the available information. 5) Decision analyses are often published in scien- 
tific journals. This prevents updating or modifying the analyses after publication, when new clinical data and medical technology inevitably become available. 6) Decision analyses are usually performed with the aid of specialist software programs that are expensive and require training. It is not easy for readers to reproduce or adjust the results. 7) Sensitivity analysis is generally deterministic and limited to one- and two-way analyses. The impact of uncertainty in the input parameters is generally not expressed in the context of variance from the other parameters in the model.

We wanted to develop a clinical decision-analysis system that would address these limitations. Using the neurosurgical example of unruptured intracranial aneurysms, we describe a computer system for decision analysis that has the following features: it is interactive for the selection of input parameters, it is applicable to individual patients, it is probabilistic, it is easy to use and understand, it can be updated rapidly, it permits interactive multi-way probabilistic sensitivity analysis, and it can be easily transmitted via the Internet.

\section{MATERIALS AND METHODS}

\section{Implementation of the System}

The system for decision analysis that we propose is a computer program written in Visual Basic for Applications in which an Excel spreadsheet is used as the interface for clinicians (Excel; Microsoft Corp., Redmond, WA). The program is available as an email attachment (write to James.Gelder@swsahs.nsw.gov.au). The program creates statistical probability distributions for estimated clinical factors from user data inputs. Feedback is provided for the user about the distributions. The program is designed to perform probabilistic simulations and the outcome of the analysis is reported with a variety of graphs and summary statistics.

\section{Decision Tree}

There are three main branches of the decision tree for unruptured intracranial aneurysms (Fig. 1). The lesion may be surgically treated, in which case the patient may die or may experience major or minor morbidity. The model described here assumes that after surgery the aneurysm is effectively obliterated and that there is no recurrence and rupture. The patient with an unruptured intracranial aneurysm may undergo observation, in which case there is a chance of rupture. If the aneurysm ruptures the patient may suffer a major or minor morbidity, or may die. If there is no rupture the patient continues to live a normal life. A patient with an unruptured intracranial aneurysm may be treated with endovascular techniques, in which case the decision-tree structure is similar to that pertaining to surgical treatment. The same assumptions about effective aneurysm obliteration and recurrence are made in the current model.

\section{User Inputs}

Initial demographic inputs include patient age, sex, and the discount rate for future life years. Inputs are required

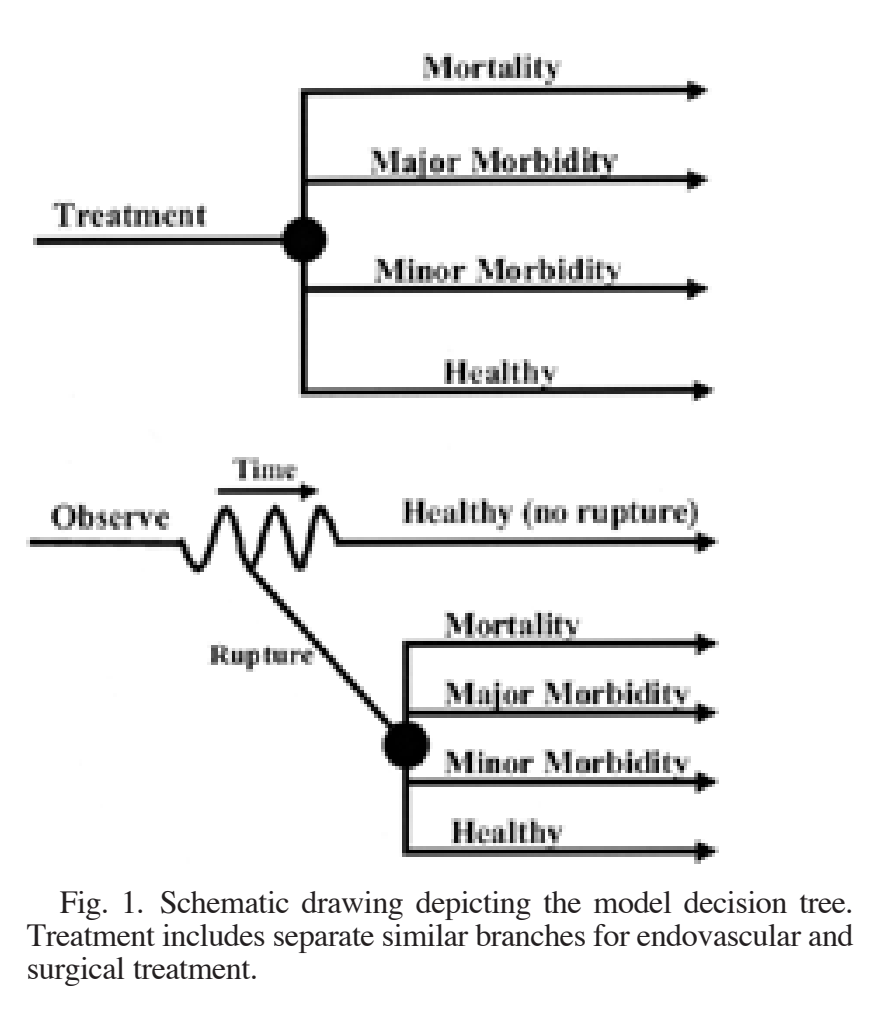

for the perceived QOL with major and minor disabilities ranging from 0 to $100 \%$. The same format is used for entering the estimated outcomes of treatment or aneurysm rupture. Clinicians are required to supply a sample size and the numbers of patients with each outcome. For example, among 150 patients five died, 10 suffered a major permanent disability, and 15 suffered a minor permanent disability. The underlined numbers are entered by the clinician. Similar methods are used for entering the estimated rate of rupture of untreated aneurysms.

\section{Calculations Related to Alternate Decisions}

The program is equipped with Monte Carlo methods to simulate outcomes for each alternate decision for 10,000 patients. The outcomes are expressed as the difference in DQALYs. Bayesian methods with noninformative conjugate prior distributions are used to derive posterior distributions for the model parameters from the input data. These include Dirichlet distributions for the outcomes of treatment or aneurysm rupture (death, minor and major disability, and normal health) and a gamma distribution for the rate of rupture.

For each simulation, parameters are sampled that describe multinomial distributions for the outcomes of treatment and rupture. A rate is sampled for the annual rate of aneurysm rupture; it is assumed that the annual rupture rate is constant over time. From these parameters discrete events are simulated for individual outcomes, such as the outcome of treatment or the time to rupture. The age at death is randomly drawn according to the Australian life table for male or female patients. This age is the same for all decision branches and represents the age at death from causes unrelated to the aneurysm. The outcome of aneurysm rupture will worsen as the patient ages. The model 
adjusts the outcome of aneurysm rupture in the future according to the age of the patient at the time of rupture. This adjustment was derived from a metaanalysis of five studies that describe the relationship between aneurysm rupture and age. ${ }^{18,19,21,26,27}$ We chose discrete event simulation rather than Markov processes, which are commonly used in medical decision analysis. This was computationally more efficient, which was particularly useful for probabilistic sensitivity analysis. We wrote code for using random-number generators using Visual Basic for Applications.

\section{Calculating the DQALYS}

For illustrative purposes we describe the case of a patient whose lesion was managed with observation and who suffered an aneurysm rupture and survived it with a major morbidity. The time to rupture (tr) is sampled from an exponential distribution, the annual discount rate is $r$, and the time to death (td) is sampled from the life table. Because the aneurysm ruptured, tr was less than td. The QOL with a major morbidity is qMajor. The formula for calculating DQALYs for this outcome is as follows:

$D Q A L Y=\int_{0}^{t r} e^{-r t} d t+q$ Major $\int_{t r}^{t d} e^{-r t} d t$

\section{Model Outputs}

The model generates DQALY values for the decision alternatives. For individual cases the program calculates the change in DQALYs between the decision alternatives. Statistical summary results include the mean, median, and 95th percentile limits for the change in DQALY values. Histograms show the distributions of the change in DQALY outcomes between the treatments. The DQALY survival curves show the percentage of patients surviving at a particular DQALY. This differs from normal survival curves, in which the percentage of patients surviving is plotted against full life years. The DQALY survival curve is identical to a standard survival curve when major and minor morbidity states are assigned a QOL of $100 \%$.

\section{Probabilistic Sensitivity Analysis}

We used a second hierarchy for the decision model applied in probabilistic sensitivity analysis (Fig. 2). In the higher level we took 1000 draws from the distributions of the parameters selected for sensitivity analysis. For each draw, in turn, we ran the standard decision analysis for 10,000 outcomes. The mean change in DQALYs was recorded. This may be regarded as a multi-way probabilistic sensitivity analysis for selected parameters. The parameters that are not selected are averaged out in the lower level of the simulation. The individual variations in the outcomes of the cases are also averaged out. The sensitivity analysis requires 10,000,000 simulated individual outcomes. The parameters used for the sensitivity analysis may be selected to be any single one or up to all of the parameters together.

\section{Expected Value of Perfect Information}

The EVPI is a summary statistic that describes the estimated cost of error in accepting the overall result of the decision analysis. ${ }^{9}$ This is provided to quantify the results

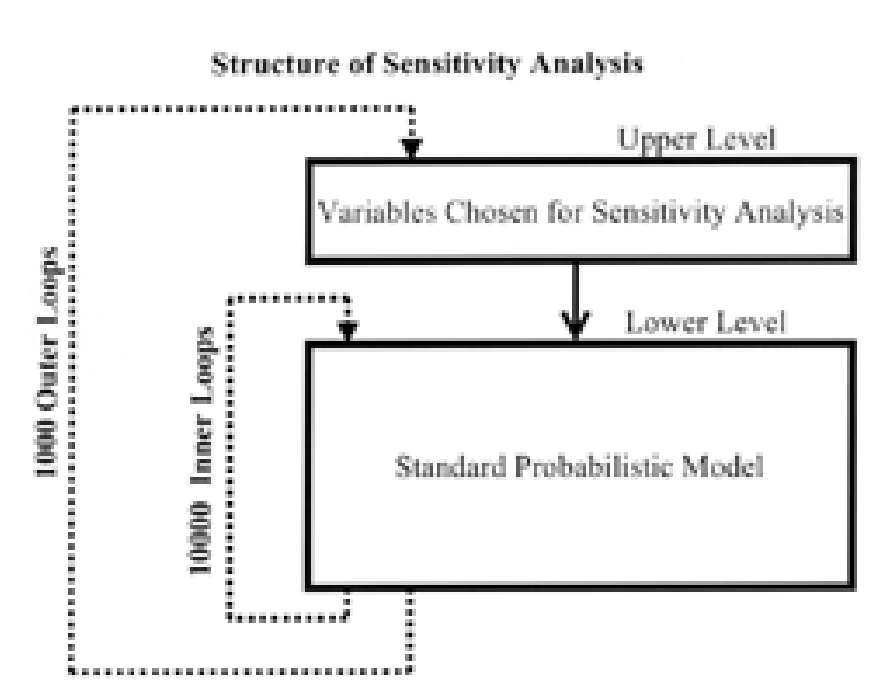

Fig. 2. Schematic drawing showing an outline of the structure of hierarchical sensitivity analysis. Variables are selected by the user for sensitivity analysis. These are randomly sampled 1000 times and held constant for each sample while the standard probabilistic model simulates outcomes for each decision for 10,000 patients.

of the probabilistic sensitivity analysis. The rationale behind the EVPI is first, that information is valuable insofar as it is likely to influence the decision, and second, that the cost of a decision is measured by the value of the next-best alternative. The mean change in DQALYs (represented by the variable $\mathrm{u}$ ) is the result of a lower-level simulation. The overall expected $\mathrm{u}$, or $\mathrm{E}(\mathrm{u})$, can be separated into a positive component (A) and a negative component (B), as is illustrated in Fig. 3.

If $\mathrm{A}+\mathrm{B}$ is positive, the decision (for example, surgery) is supported by the model and the EVPI is $-\mathrm{B}$. Further information that increases the expected value of $\mathrm{A}$ will not change the optimum decision. If $\mathrm{A}+\mathrm{B}$ is negative, the alternative (for example, observation) is supported and the EVPI is A.

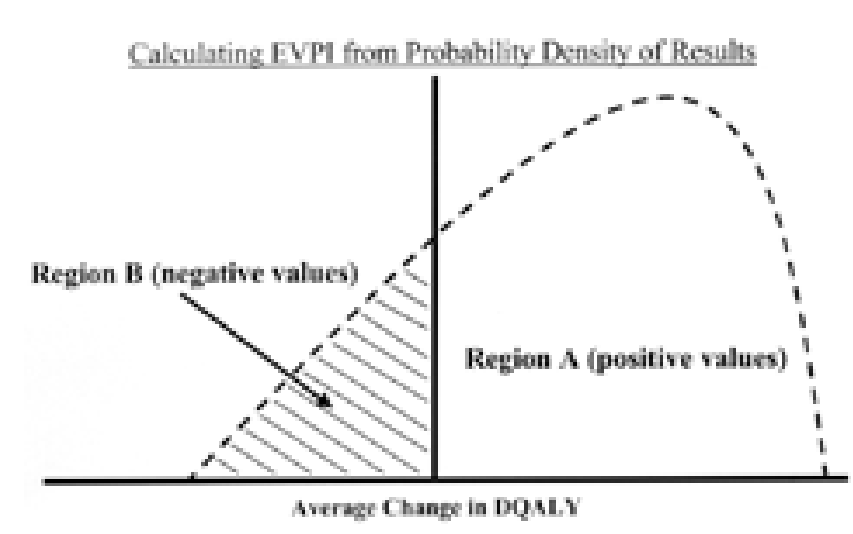

Fig. 3. Schematic figure illustrating the principle of the EVPI. The probability density is graphed against the mean change in DQALYs for a comparison of two treatments. Variance arises from the parameters selected for sensitivity analysis. If the overall result of the model is positive, Region B is the EVPI, and if the overall result of the model is negative, Region A is the EVPI. 


\section{RESULTS}

The data for a sample simulation are presented in Table 1 and the results are in Table 2. This contains information on a 45-year-old woman without risk factors or comorbidities who had a $10-\mathrm{mm}$ anterior circulation aneurysm and no history of treatment for ruptured lesions. Estimates are derived from the International Study of Unruptured Intracranial Aneurysms. ${ }^{30}$ The data used for input and distributions for the aneurysm rupture rate, the outcome of treatment, and the outcome of aneurysm rupture used for the simulations are specified in Table 1.

The illustrative case is presented with data from a single source that is widely available. This data source has been selected only for the purpose of illustration of the model and we do not wish to endorse or criticize the study. The DQALY survival plots are shown in Fig. 4 for the endovascular, surgical, and observation treatment decisions. The QOL considerations for patients with major or minor disabilities are important interactive parameters that reflect the values of the decision maker or the patients. Separate DQALY survival plots are shown in which $0 \%, 25 \%$, and $100 \%$ are assumed for the QOL (Fig. 4). For this illustrative case the model predicts an initial survival advantage for the observation arm that is not significantly overcome by the delayed benefits of either the endovascular or surgical options for the remainder of the individual's life. A comparison of surgery and endovascular strategies is also shown in the change in the DQALY histogram (Fig. 5). This illustrates that for most simulated outcomes there is no change in DQALYs between undergoing endovascular and receivin surgical treatments.

For this example, in which $25 \%$ is used for the QOL with morbidity, surgery compared with endovascular treatment has a mean predicted DQALY change of 1.3 favoring surgery (95\% CI -18 to 24.8 ; Table 2 ). For surgery compared with observation, the mean predicted DQALY change was 0 , implying no strong trend in either direction

TABLE 1

Input data for the illustrative case of a patient with an unruptured intracranial aneurysm*

\begin{tabular}{lrl}
\hline \hline \multicolumn{1}{c}{ Parameter } & Input & \multicolumn{1}{c}{ Distribution } \\
\hline age (yrs), sex & $45, \mathrm{~F}$ & life table \\
discount & $3 \%$ & \\
op outcome & & Dirichlet $(5,17,381)$ \\
$\quad$ death & 4 & $1 \%(0.3-2.2 \%)$ \\
disability & 16 & $4 \%(2.3-6.1 \%)$ \\
sample size & 400 & \\
endo outcome & & Dirichlet $(3,4,66)$ \\
death & 2 & $2.9 \%(0.4-8.1 \%)$ \\
disability & 3 & $4.3 \%(0.9-10 \%)$ \\
sample size & 70 & Dirichlet $(3,8,13)$ \\
rupture outcome & & $9.6 \%(1.2-25 \%)$ \\
death & 2 & $33.1 \%(14.8-54 \%)$ \\
disability & 7 & gamma $(4,0.002)$ \\
sample size & 21 & $0.6 \%(0.2-1.3 \%)$ \\
annual rupture rate & & \\
no. of ruptures & 3 & \\
patient-yrs & 650 & \\
\hline
\end{tabular}

* The decision support system provides options of major and minor disability, but in the study by Wiebers, et al., only one level of disability was described. Abbreviation: endo = endovascular.
TABLE 2

Simulation results in the illustrative case of a patient with an unruptured intracranial aneurysm*

\begin{tabular}{lccc}
\hline \hline & \multicolumn{3}{c}{ QOL } \\
\cline { 2 - 4 } Change in DQALY & $25 \%$ & $0 \%$ & $100 \%$ \\
\hline op minus endo mean & 1.3 & 1.1 & 2 \\
95\% CI & -18 to 24.8 & -23.1 to 24.8 & 0 to 24.8 \\
median & 0 & 0 & 0 \\
op minus obs mean & 0 & -0.2 & 0.5 \\
95\% CI & -17.9 to 13.4 & -23.1 to 14.4 & 0 to 11.7 \\
median & 0 & 0 & 0 \\
endo minus obs mean & -1.3 & -1.3 & -1.5 \\
95\% CI & -24.7 to 13 & -24.5 to 13.4 & -24.7 to 11 \\
median & 0 & 0 & 0 \\
\hline
\end{tabular}

$*$ Obs = observation.

(95\% CI -17.9 to 13.4 ). For endovascular compared with observation treatment, the mean predicted DQALY change was -1.3 favoring observation $(95 \%$ CI -24.7 to 13). These data and the model indicate that observation and surgery are both preferable to endovascular treatment. The confidence limits represent 2.5 and 97.5 th percentiles for individual simulated cases. These limits represent more extreme cases such as death due to treatment or death due to early aneurysm rupture while the patient was under observation.

\section{Probabilistic Sensitivity Analysis}

A probabilistic sensitivity analysis was performed for the parameters surgical outcome, rupture outcome, and rupture rate for the sample cases in which we compared observation and surgery (Fig. 6). The variance in the mean change in DQALYs is most sensitive to the variance in the estimated rate of rupture because of the small sample size of the data source. Furthermore, within the range of predicted mean change in DQALYs lies strong support for both surgery and observation. In contrast, the variance in change in DQALYs is less sensitive to the outcome of rupture and of surgery. Further data about the rate of rupture of these aneurysms will have a pronounced effect on the confidence in the results of the model. Further data on the outcomes of aneurysm treatment and aneurysm rupture will have a smaller effect on the confidence in the results of the model. It is notable that the variance in the all-parameter analysis is similar to that in the rupture rate analysis. This further demonstrates the importance of the rupture rate to the overall variance of the model. The EVPI predictions are given in Table 3 . These provide a quantitative estimate of the variance in the output resulting from one or more variables. The highest EVPIs are associated with the variance in the annual rupture rate (Table 3).

\section{DISCUSSION}

This tool addresses the limitations of published clinical decision analyses that were identified earlier. Our decision-analysis system can be adapted to individual patients. The inputs can be derived from large studies or specifical- 

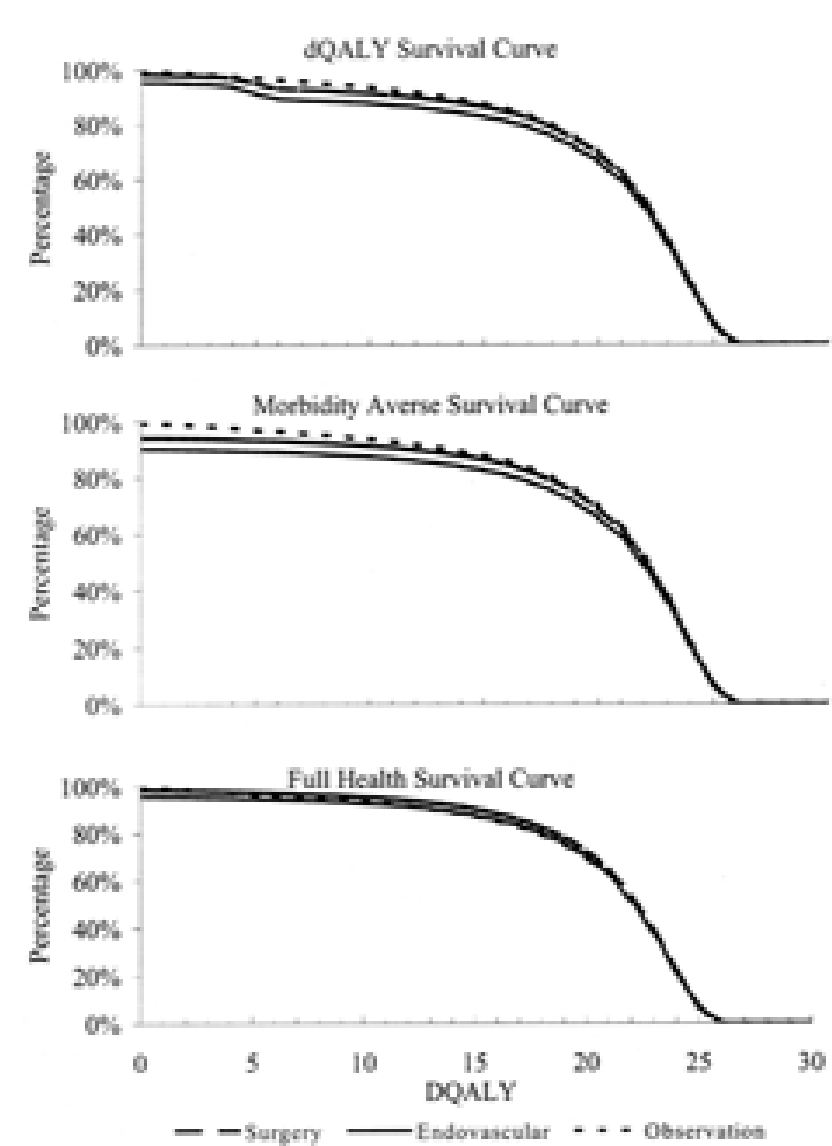

Fig. 4. Graphs depicting DQALY survival plots for the simulated outcome of both treatment and observation for the illustrative case described in Table 1. It is important to note that the $\mathrm{x}$ axis represents DQALYs and not years. For this example the immediate cost of treatment compared with observation is never overcome by the delayed benefit. The rapid falloff after 20 years is caused by the increasing effects of discounting and the shape of the life expectancy distribution. In the upper panel we have used an intermediate value for QOL for morbidity of $25 \%$. In the center panel we have used a QOL of $0 \%$ for morbidity, representing a morbidity-averse patient who places value only on full health and independence. In the lower panel we have used a QOL for morbidity of 100\%, representing a death-averse patient who places value on life at all costs. The decision to treat is similar in outcome for the intermediate and death-averse patients. The morbidity-averse patients would be likely to choose observation rather than treatment.

ly adjusted or selected for particular patients by the use of expert opinion. The values for QOL with morbidity and the discount rate can be adjusted according to the preferences of the patient. The model can be updated or improved and distributed over the Internet. Graphic presentations of the results complement summary statistics such as the mean change in DQALYs. Multiple methods are available for interactive sensitivity analysis.

We are not aware of any comparable fully probabilistic and interactive medical decision-analysis systems that are accessible on the Internet. We originally conceived this project as an Internet site that would perform Monte Carlo simulations and return the results. The ubiquitous availability of Microsoft Excel and the advantages of using it as the interface for producing graphs were fac-

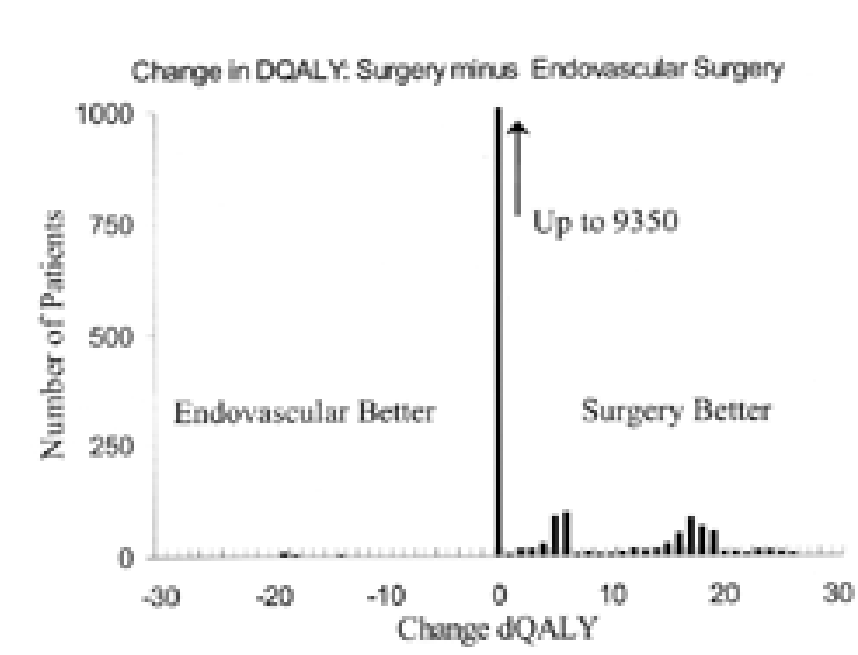

Fig. 5. Histogram showing the distribution of the change in DQALYs (surgery DQALYs - endovascular DQALYs). The most frequent value for change in DQALYs is 0 and is off the scale. This is to be expected because most aneurysms would not rupture under observation and most treatments for unruptured aneurysms would not cause morbidity or death. For the majority of patients the decision will not influence their quality or length of life.

tors that caused us to write the program with Visual Basic for Applications. We are unaware of any previous use of DQALY survival graphs and histograms to express the results of simulations of thousands of patients. We are also unaware of any previous use of hierarchical simulations for probabilistic sensitivity analysis in medical decision models.

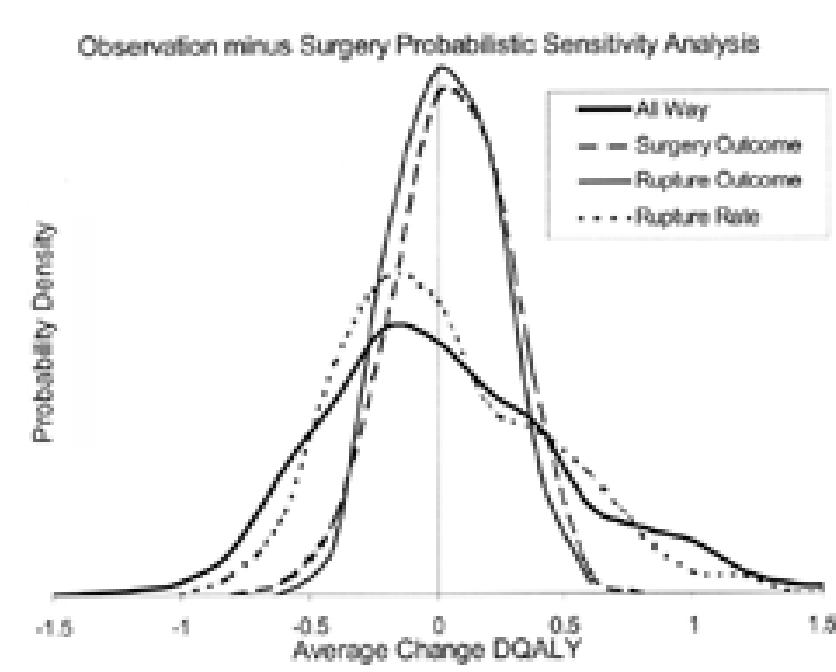

Fig. 6. Graph showing the probabilistic sensitivity simulation results for comparing observation and surgery. The sensitivity analysis was performed separately for surgical outcome, rupture outcome, rupture rate, and all-way analysis (all of these factors combined). The rupture rate analysis generated the most variance (the widest distribution) for the mean change in DQALYs of any individual factor. To improve the overall confidence of the model results, the variance has to be reduced. Because the rupture rate contributes most to the variance, more data about this parameter will improve the confidence the most. 
TABLE 3

Sensitivity analysis assessing the EVPI in patients with unruptured intracranial aneurysms*

\begin{tabular}{lccccc}
\hline \hline & \multicolumn{5}{c}{ Parameter } \\
\cline { 2 - 6 } Comparison & All-Way & $\begin{array}{c}\text { Op } \\
\text { Outcome }\end{array}$ & $\begin{array}{c}\text { Endo } \\
\text { Outcome }\end{array}$ & $\begin{array}{l}\text { Rupture } \\
\text { Outcome }\end{array}$ & $\begin{array}{c}\text { Rupture } \\
\text { Rate }\end{array}$ \\
\hline endo vs obs & 0.493 & NA & 0.138 & 0 & 0.296 \\
op vs obs & 0.426 & 0.151 & NA & 0.130 & 0.370 \\
op vs endo & 0.158 & 0 & 0.152 & NA & NA \\
\hline
\end{tabular}

* The EVPI is expressed in the units of DQALY. Abbreviation: NA = not applicable.

\section{Data Entry}

Users are required to enter estimates of major parameters for the decision model as probability distributions. Some users may not be familiar with statistical methods for deriving posterior distributions for parameters from clinical data or expert opinion. To produce a simple and consistent method for extracting distributions we ask users to provide the estimated parameter and the sample size. The program provides feedback on Bayesian posterior distributions, including the mean and 95\% limits. We are not aware of any previous use of this method in interactive decision support systems. We have found in trials of this program that when they are unsure, users frequently enter a sample size of 100 , from which the program derives the variance of the parameter. Where distributions are symmetrical, the variance will not affect the mean of the simulated outcomes. For asymmetrical distributions, the variance will affect the mean of simulated outcomes. Asymmetrical distributions are possible with small sample sizes. In contrast, the variance of the parameters will have a large effect on the probabilistic sensitivity analysis.

\section{Sensitivity Analysis}

Elementary one-way sensitivity analysis can be performed by varying any of the inputs and rerunning the simulation. Performed in this way, the importance of systematic bias in the estimates of parameters may be evaluated. This can be refined further to a one-way probabilistic sensitivity analysis by entering data normally for the parameter of interest and reducing all other parameters to virtual point estimates by artificially increasing their sample size. In this case, variance in the output will reflect both the variance from the parameter of interest and the individual outcomes for the simulated cases (such as variance from the actual time to aneurysm rupture or the variance from the life expectancy). A more sophisticated sensitivity analysis may be performed by using the multi-way hierarchical probabilistic sensitivity analysis facility. In this case the variance from the unselected parameters and the variance from individual outcomes is averaged out in the lowest level of the simulations. The higher level of the simulation then provides a distribution for the mean change in DQALYs. The variance in this distribution arises from a variance in the selected parameters for sensitivity analysis. Performed in this way, the importance of variance arising from small sample sizes or imprecise estimates can be explored. The user may examine the effect of one parameter selected, or he/she may examine the effects of removing one parameter at a time from the selected variables.

\section{Expected Value of Perfect Information}

The EVPI may be used to gauge the importance of variance in the estimates of the input parameters. When calculated for individual parameters it may be used to assess and compare the sensitivity of the model to the variance from each parameter. The EVPI quantifies the impact that further research into the selected parameters may potentially have on the decision. In the example described in this study, the EVPI for the aneurysm rupture rate substantially exceeds the EVPI for the outcome of aneurysm rupture and treatment. This indicates that further research into the annual rate of aneurysm rupture will be likely to have the greatest influence on the decision analysis. Further information is likely to affect significantly either one or both of the following: the results of the model and/or the confidence that may be placed in the results.

Further research on the morbidity and mortality rates related to aneurysm treatment will have a smaller influence. The EVPI is sensitive to systematic bias in the estimation of the input parameters. In this case the EVPI will be systematically biased. The effects of possible systematic bias are best assessed by one-way or two-way sensitivity analysis. A second interpretation of the EVPI is not illustrated here. The EVPI with all parameters selected may be contrasted with the EVPI with all selected except the parameter of interest, which is averaged out in the lower level of the simulation. This method produces the estimated change in overall EVPI with improved knowledge of the parameter of interest.

\section{Complexity of the Decision}

The decision to treat unruptured aneurysms is complex and depends on a number of separate sources of information as well as expert application of the information to individual cases. It is evident that quantitative calculations will assist rational decision making. In medical decision analysis, complex problems are divided into component pathways or branches. Risks are quantified, and a common utility is defined to compare outcomes with different life expectancies and QOLs. We have tried to present a model that incorporates the most important factors without becoming overly complex and alienating clinical users. Therefore, for this model we make a number of assumptions. A more complex model is technically easy to produce but would necessarily ask the user for inputs for which there is little meaningful data or expert opinion. Some important complexities that we have refrained from incorporating into the model are listed here.

1. With this model we assume a constant rate of rupture over time, and there is some evidence for this in the survival plots of observational studies. ${ }^{14,31}$ Alternatively, it has been proposed that aneurysms may have an early unstable period. ${ }^{22}$ If the aneurysm rupture rate decreases over time, then the model will be biased toward treatment.

2. The model does not adjust the DQALYs for time spent undergoing treatment of aneurysms or recovering from ruptures. ${ }^{13,25}$ This will bias the model toward aneurysm treatment. 
Interactive, probabilistic decision analysis in unruptured aneurysm

3. The model does not include rupture of aneurysms after treatment. There is a small risk of rupture after treatment with both clip placement and endovascular coil occlusion. ${ }^{8,28,29}$ This will bias the model toward treatment.

4. The model does not incorporate a joint distribution correlating the outcome of rupture and the outcome of treatment. The user may simultaneously enter a high morbidity rate associated with both rupture and treatment for an individual patient because of known risk factors. Nevertheless, a high-risk patient in whom surgery is considered may also have a high risk of poor outcome from aneurysm rupture.

5. The model falls short of explicitly depicting many complex clinical scenarios. For example, there is currently no decision option for observing an aneurysm and treating it if it becomes larger. ${ }^{16}$ There is also no capacity to model multiple aneurysms. These factors will bias the model toward early treatment. The model does not adjust the QALYs in the observation arm for the anxiety of living with an untreated aneurysm. This will bias the model toward observation. There is no capacity to incorporate the possibility for subsequent retreatment with coil occlusion or surgery. ${ }^{4}$ There is no capacity for the model to incorporate the subsequent development of symptoms such as embolic events. ${ }^{24}$ There is no capacity to model future improvements in technology. These last three would also bias the model toward observation.

6 . The present model does not incorporate the projected financial costs of each outcome into a cost-effectiveness study.

\section{Intended Use of the Model}

We have not intended that the model be used to prescribe a decision choice for an individual. We expect the model to provide a framework for which an expert opinion is required for the final recommendation. The model may increase the confidence of the patient or the decision maker in their final decision.

\section{Sources of Information}

We advise great care and experienced judgment in extracting data from clinical studies for application to a specific patient. Clinical studies may describe a treatment series rather than a management series, and therefore may provide limited information on the selection of patients for treatment. Publication bias arises from the difference between clinical experiences and published clinical studies that often present small groups of patients or surprising or favorable findings. Publication bias may cause a bias toward treatment. Technological differences between the reported studies and the locally available methods and expertise may cause a bias toward treatment. Clinical studies usually present summaries of information that may not address the precise clinical question being posed or may not provide enough detailed stratification of the participants to enable the data to be applied to a specific patient. Studies of the outcome in patients with ruptured intracranial aneurysms usually describe hospital-based series rather than the results for a whole population. ${ }^{11}$ These studies usually provide outcomes stratified by clinical features such as the level of consciousness at presentation with rupture. In contrast the important clinical features the age and the na- ture of the aneurysm when modeling decisions for patients with unruptured aneurysms.

\section{Alternative Uses}

The decision structure shown in Fig. 1 is not restricted to use in unruptured intracranial aneurysms. We envisage the model or its adaptations being used with other medical interventions that can be applied to asymptomatic individuals to prevent a catastrophic event that might otherwise occur.

\section{Further Research}

It has previously been shown that decision-tree methods and decision support systems may be useful in clinical practice for both clinicians and patients. ${ }^{3,23}$ Critical factors include the provision of information, speed and accessibility, simplicity, and adaptability. ${ }^{3}$ Further research is required to determine whether the form of decision analysis presented here will be useful in clinical practice, particularly because we are unaware of any similar medical projects. ${ }^{17}$ It remains to be shown whether the system will influence decision making, or whether it will have any significant use in the development of general policy, for application to individual cases, for communicating with patients, or for planning the most efficient medical research.

Further research is required into the appropriate level of complexity of the model. Further work is required in the critical appraisal of the literature and preparation of information for estimating patient-specific parameters. Further research is required to determine the best method for practitioners to express clinically uncertain parameters and to determine the best expression of the results of the simulations for human decision making.

\section{CONCLUSIONS}

We hope that the availability of models such as this will provide feedback to clinical researchers in the following ways: 1) by identifying the uncertain factors that have the greatest potential leverage on clinical decisions; 2) by defining the research questions for the quantitative decision maker; and 3) by showing the need for more detail and stratification of the results of clinical research.

\section{References}

1. Aoki N, Kitahara T, Fukui T, et al: Management of Unruptured intracranial aneurysm in Japan: a Markovian decision analysis with utility measurments based on the Glascow Outcome Scale. Med Decis Making 18:357-364, 1998

2. Aoki N, Beck JR, Kitahara T, et al: Reanalysis of unruptured intracranial aneurysm management: effect of a new international study on the threshold probabilities. Med Decis Making 21: 87-96, 2001

3. Bates DW, Kuperman GJ, Wang S, et al: Ten commandments for effective clinical decision support: making the practice of evidence-based medicine a reality. J Am Med Inform Assoc 10:523-530, 2003

4. Bavinzski G, Talazoglu V, Killer M, et al: Coiling of recurrent and residual cerebral aneurysms after unsuccessful clipping. Minim Invasive Neurosurg 42:22-26, 1999

5. Butler WE, Barker FG II, Crowell RM: Patients with polycystic kidney disease would benefit from routine magnetic resonance 
angiographic screening for intracerebral aneurysms: a decision analysis. Neurosurgery 38:506-516, 1996

6. Dippel DW, ter Berg JW, Habbema JD: Screening for unruptured familial intracranial aneurysms: a decision analysis. Acta Neurol Scand 86:381-389, 1992

7. Dolan JG: Clinical decision analysis. Med Decis Making 21: 150-151, 2001

8. Eskridge JM, Song JK: Endovascular embolization of 150 basilar tip aneurysms with Guglielmi detachable coils: results of the Food and Drug Administration multicenter clinical trial. J Neurosurg 89:81-86, 1998

9. Felli JC, Hazen GB: Sensitivity analysis and the expected value of perfect information. Med Decis Making 18:95-109, 1998

10. Gaetani P, Rodriguez y Baena R, Klersy C, et al: A cost-effectiveness analysis on different surgical strategies for intracranial aneurysms. J Neurosurg Sci 42:69-78, 1998

11. Huang J, van Gelder JM: The probability of sudden death from rupture of intracranial aneurysms: a meta-analysis. Neurosurgery 51:1101-1107, 2002

12. Inomiya K, Sakurai T, Kaihara S: Effectiveness of preventive surgery for asymptomatic unruptured intracranial aneurysms. Medinfo 8:889-893, 1995

13. Johnston SC, Gress DR, Kahn JG: Which unruptured cerebral aneurysms should be treated? A cost-utility analysis. Neurology 52:1806-1815, 1999

14. Juvela S, Porras M, Poussa K: Natural history of unruptured intracranial aneurysms: probability of and risk factors for aneurysm rupture. J Neurosurg 93:379-387, 2000

15. Kallmes DF, Kallmes MH, Cloft HJ, et al: Guglielmi detachable coil embolization for unruptured aneurysms in nonsurgical candidates: a cost-effectiveness exploration. AJNR 19:167-176, 1998

16. Kamitani H, Masuzawa H, Kanazawa I, et al: Bleeding risk in unruptured and residual cerebral aneurysms-angiographic annual growth rate in nineteen patients. Acta Neurochir 141: 153-159, 1999

17. Kaplan B: Evaluating informatics applications-clinical decision support systems literature review. Int J Med Inform 64: 15-37, 2001

18. Kassell NF, Torner JC, Haley EC Jr, et al: The International Cooperative Study on the Timing of Aneurysm Surgery. Part 1: Overall management results. J Neurosurg 73:18-36, 1990

19. Lanzino G, Kassell NF, Germanson TP, et al: Age and outcome after aneurysmal subarachnoid hemorrhage: why do older patients fare worse? J Neurosurg 85:410-418, 1996

20. Leblanc R, Worsley KJ: Surgery of unruptured, asymptomatic aneurysms: a decision analysis. Can J Neurol Sci 22:30-35, 1995
21. Ljunggren B, Saveland H, Brandt L, et al: Aneurysmal subarachnoid hemorrhage. Total annual outcome in a 1.46 million population. Surg Neurol 22:435-438, 1984

22. Mitchell P, Jakubowski J: Risk analysis of treatment of unruptured aneurysm. J Neurol Neurosurg Psychiatry 68:577-580, 2000

23. O'Connor AM, Mulley AG Jr, Wennberg JE: Standard consultations are not enough to ensure decision quality regarding preference-sensitive options. J Natl Cancer Inst 95:570-571, 2003

24. Qureshi AI, Mohammad Y, Yahia AM, et al: Ischemic events associated with unruptured intracranial aneurysms: multicenter clinical study and review of the literature. Neurosurgery 46: 282-290, 2000

25. Raaymakers TW: Functional outcome and quality of life after angiography and operation for unruptured intracranial aneurysms. On behalf of the MARS Study Group. J Neurol Neurosurg Psychiatry 68:571-576, 2000

26. Rosenorn J, Eskesen V, Schmidt K: Age as a prognostic factor after intracranial aneurysm rupture. Br J Neurosurg 1: 335-341, 1987

27. Saveland H, Brandt L: Which are the major determinants for outcome in aneurysmal subarachnoid hemorrhage? A prospective total management study from a strictly unselected series. Acta Neurol Scand 90:245-250, 1994

28. Tsutsumi K, Ueki K, Usui M, et al: Risk of subarachnoid hemorrhage after surgical treatment of unruptured cerebral aneurysms. Stroke 30:1181-1184, 1999

29. Tsutsumi K, Ueki K, Morita A, et al: Risk of aneurysm recurrence in patients with clipped cerebral aneurysms: results of long-term follow-up angiography. Stroke 32:1191-1194, 2001

30. Wiebers DO, Whisnant JP, Huston J III, et al: Unruptured intracranial aneurysms: natural history, clinical outcome, and risks of surgical and endovascular treatment. Lancet 362:103-110, 2003

31. Yasui N, Suzuki A, Nisimura H, et al: Long-term follow-up study of unruptured intracranial aneurysms. Neurosurgery 40: 1155-1160, 1997

32. Yoshimoto Y, Wakai S: Cost-effectiveness analysis of screening for asymtomatic, unruptured intracranial aneurysms. A mathematical model. Stroke 30:1621-1627, 1999

Manuscript received September 16, 2004.

Accepted in final form October 5, 2004.

Address reprint requests to: James M. van Gelder, M.D., Department of Neurosurgery, Liverpool Health Service, Liverpool, New South Wales, 2170 Australia. email: James.Gelder@swsahs.nsw.gov.au. 